\title{
S-shooting: a Bennett-Chandler-like method for the computation of rate constants from committor trajectories
}

\author{
Georg Menzl, Andreas Singraber and Christoph Dellago*
}

Received 9th May 2016, Accepted 14th June 2016

DOI: $10.1039 / c 6 f d 00124 f$

\begin{abstract}
Mechanisms of rare transitions between long-lived stable states are often analyzed in terms of commitment probabilities, determined from swarms of short molecular dynamics trajectories. Here, we present a computer simulation method to determine rate constants from such short trajectories combined with free energy calculations. The method, akin to the Bennett-Chandler approach for the calculation of reaction rate constants, requires the definition of a valid reaction coordinate and can be applied to both under- and overdamped dynamics. We verify the correctness of the algorithm using a one-dimensional random walker in a double-well potential and demonstrate its applicability to complex transitions in condensed systems by calculating cavitation rates for water at negative pressures.
\end{abstract}

\section{Introduction}

Many processes occurring in molecular systems are dominated by rare transitions between long-lived states. ${ }^{1,2}$ Examples include nucleation during first-order phase transitions, chemical reactions in solution, and conformational changes of biological macromolecules. Understanding the molecular mechanism of such transitions is challenging due to the large number of interacting degrees of freedom and the resulting complex collective behavior. In analyzing rare transitions in complex systems, the goal is to find a reaction coordinate, i.e., a dynamically meaningful variable that captures the essential physics of the transition and is capable of quantifying its progress. Once a reaction coordinate is known it may be used to construct low-dimensional mechanistic models ${ }^{3,4}$ and, furthermore, enhance the sampling of transition pathways and the calculation of rate constants. $^{5-8}$

A good reaction coordinate should be able to tell us what is likely to happen next. Hence, the quality of a reaction coordinate can be assessed in terms of the committor, i.e., the probability of a given configuration to first reach the product 
rather than the reactant region. In fact, it has been noted ${ }^{9-11}$ that the committor itself is the perfect reaction coordinate since, by its very definition, it measures the progress of reaction. However, knowledge of the committor as a function of the configuration does not automatically yield any insight into the nature of the collective variables that are relevant for the transition. Nevertheless, the committor is very useful in the analysis of reaction mechanisms, because it permits to test reaction coordinates postulated based on physical reasoning or on the analysis of reactive trajectories. ${ }^{12,13}$ Also, several methods for the automatic extraction and optimization of reaction coordinates based on the committor have been proposed. ${ }^{\mathbf{9}, \mathbf{1 4 - 1 7}}$

From a good reaction coordinate one expects that its value completely determines the committor, or, in other words, that iso-surfaces of the reaction coordinate are also iso-surfaces of the committor. Whether this is the case can be tested by computing the committor for configurations with a given value of the reaction coordinate. This can be done by initiating multiple short trajectories from these configurations and counting how many of them reach the product state before the reactant state. Frequently, such calculations are combined with an estimate of the free energy as a function of the reaction coordinate, which, provided the reaction coordinate reflects the underlying mechanism, provides information on the nature of the transition state, i.e., of the dynamical bottleneck the system needs to cross during the transition. If committor calculations are carried out also near the transition state region, the dynamical information obtained from the fleeting trajectories can be combined with the free energy landscape to determine rate constants for the transition, as has been recently suggested by Daru and Stirling. ${ }^{18}$ Their divided saddle theory, which may be viewed as generalization of the celebrated Bennett-Chandler method for the calculation of rate constants, ${ }^{19,20}$ provides an efficient way to determine rate constants by post-processing information harvested in free energy and committor calculations.

In this article, we present an alternative way to extract reaction rate constants from committor trajectories and the free energy profile. Like the BennettChandler method and the divided saddle theory, the method is based on a factorization of the rate constant expression into two factors, one that can be expressed in terms of the free energy and another one that contains dynamical information. In our approach, the factorization is applied on the level of the time correlation function of the populations of the stable states between which the transition occurs. From the linear regime of this correlation function the rate constant is then extracted. Since the time correlation function is considered instead of the reactive flux, which requires a well-defined time-derivative of the reaction coordinate at the interface, the method can be applied equally well to the under- and over-damped case. The shape of the time correlation function, which is evaluated from the committor trajectories, provides additional insight into the barrier crossing dynamics. In the following, we will first outline the algorithm (details of the derivation are provided in the Appendix) and then demonstrate the application of the method to two test cases: a Brownian walker in a one-dimensional double well potential and cavitation of water at negative pressures. 


\section{S-shooting algorithm}

The goal of the algorithm presented here is to determine the rate constant of transitions between two long-lived states, A and B, which can be viewed as the reactant and the product state, respectively. We assume that we are able to distinguish between these two states using a reaction coordinate $q(x)$ that tracks the progress of the transition, i.e., a suitable collective variable defined for each microscopic configuration $x$. In practice, $q(x)$ can vary considerably in its complexity depending on the investigated system: it can be as simple as a Cartesian coordinate in cases where the underlying (free) energy landscape is known (as is the case for a Brownian walker in a double well potential in Section 3.1) or can be based upon detecting the largest cluster of a nucleating phase in the case of first-order phase transitions (see Section 3.2).

\subsection{Time correlation function and reaction rate constant}

The method presented here is rooted in the Bennett-Chandler approach, ${ }^{\mathbf{1 9}, 20}$ in which the transition rate constant is expressed in terms of the time correlation function of the populations of the stable states. To introduce this correlation function, we first define the characteristic functions for states A and B, which indicate if the system is in the respective state or not,

$$
h_{\mathrm{A}}(x)= \begin{cases}1 & \text { if } x \in A \\ 0 & \text { else }\end{cases}
$$

and $h_{\mathrm{B}}(x)$ is defined analogously for region B. In all cases considered in this work, the underlying free energy landscapes determining the behavior of the systems exhibit a barrier dividing the two states. We assume that the regions $\mathrm{A}$ and $\mathrm{B}$ correspond to the ranges $\mathrm{A}=\left(-\infty, q_{\mathrm{A}}\right)$ and $\mathrm{B}=\left(q_{\mathrm{B}}, \infty\right)$ of the reaction coordinate, respectively, and $q_{\mathrm{A}}<q_{\mathrm{B}}$. Accordingly, we define the characteristic functions as $h_{\mathrm{A}}(x)=1-\theta\left[q(x)-q_{\mathrm{A}}\right]$ and $h_{\mathrm{B}}(x)=1-\theta\left[q_{\mathrm{B}}-q(x)\right]$, where $\theta(q)$ is the Heaviside step function. The time correlation function

$$
C_{\mathrm{AB}}(t)=\frac{\left\langle h_{\mathrm{A}}(0) h_{\mathrm{B}}(t)\right\rangle}{\left\langle h_{\mathrm{A}}\right\rangle}
$$

encodes the conditional probability to find the system in B at time $t$ provided it was in $\mathrm{A}$ at time 0 . In the above expression, $h_{\mathrm{B}}(t)$ is a shorthand for $h_{\mathrm{B}}\left(x_{t}\right)$, where $x_{t}$ is the microscopic state of the system at time $t$, and the angular brackets $\langle\ldots\rangle$ denote equilibrium averages. The equilibrium probability of finding the system in A can be expressed as

$$
\left\langle h_{\mathrm{A}}\right\rangle=\frac{\int \mathrm{d} x \mathrm{e}^{-\beta H(x)} h_{\mathrm{A}}(x)}{\int \mathrm{d} x \mathrm{e}^{-\beta H(x)}}=\int_{-\infty}^{q_{\mathrm{A}}} \mathrm{d} q \mathrm{e}^{-\beta F(q)},
$$

where $\beta=1 / k_{\mathrm{B}} T$ with the Boltzmann constant $k_{\mathrm{B}}$ and temperature $T$, and $H(x)$ is the total energy of the system. The free energy $F(q)$ is related to the probability density $p(q)=\langle\delta[q-q(x)]\rangle$ of the reaction coordinate by $F(q)=-k_{\mathrm{B}} T \ln p(q)$. After initial transient behavior related to the details of the dynamics and the specific definition of the stable states, the time correlation function $C_{\mathrm{AB}}(t)$ is expected to enter a linear regime and its time derivative gives the reaction rate constant $k_{\mathrm{AB}}$, 


$$
k_{\mathrm{AB}}=\frac{\mathrm{d} C_{\mathrm{AB}}(t)}{\mathrm{d} t} .
$$

The rate constant $k_{\mathrm{BA}}$ for the inverse reaction from $\mathrm{B}$ to $\mathrm{A}$ is obtained by applying detailed balance, $k_{\mathrm{BA}}=k_{\mathrm{AB}}\left\langle h_{\mathrm{A}}\right\rangle /\left\langle h_{\mathrm{B}}\right\rangle$. Together, the forward and backward rate constants determine the reaction time $\tau_{\mathrm{rxn}}=\left(k_{\mathrm{AB}}+k_{\mathrm{BA}}\right)^{-1}$, the time scale at which a non-equilibrium population of states $\mathrm{A}$ and $\mathrm{B}$ decays to equilibrium.

\subsection{S-ensemble}

The S-shooting algorithm presented here introduces an additional region, S, located such that any trajectory transitioning from A to B must cross S (see Fig. 1). As illustrated in the figure, there are various possibilities to define region $\mathrm{S}$, which plays essentially the same role as the saddle region in divided saddle theory. ${ }^{18}$ If A and $\mathrm{B}$ are adjacent and separated by a dividing surface, $\mathrm{S}$ could be located entirely in A or B or include parts of both regions. In this case, it is only required that the dividing surface is part of $\mathrm{S}$. If regions $\mathrm{A}$ and $\mathrm{B}$ are not adjacent but rather separated from each other, region S can be in A or B or somewhere in between. The only requirement is that no trajectory can connect A with B without visiting $\mathrm{S}$. Although the particular definition of $S$ does not affect the validity of the expressions we will derive in the following, its particular location will have an effect on the statistical accuracy of the rate constant estimation. To make the rate calculation efficient, region S should include the transition state region, from which both stable states are accessible with non-vanishing probability.

Since any trajectory which gives a non-zero contribution to the correlation function $C_{\mathrm{AB}}(t)$ has at least one configuration in $\mathrm{S}$ by construction, it should be possible to express $C_{\mathrm{AB}}(t)$ as path average in the ensemble of trajectories touching $\mathrm{S}$. We call this ensemble of trajectories the S-ensemble. In the following we consider discretized trajectories $x(\tau)=\left\{x_{0}, x_{1}, x_{2}, \cdots, x_{\mathrm{L}}\right\}$ with fixed length $\tau=L \Delta t$ consisting of $L+1$ configurations separated by the time step $\Delta t$. Such trajectories may result, for instance, from a molecular dynamics simulation. The probability
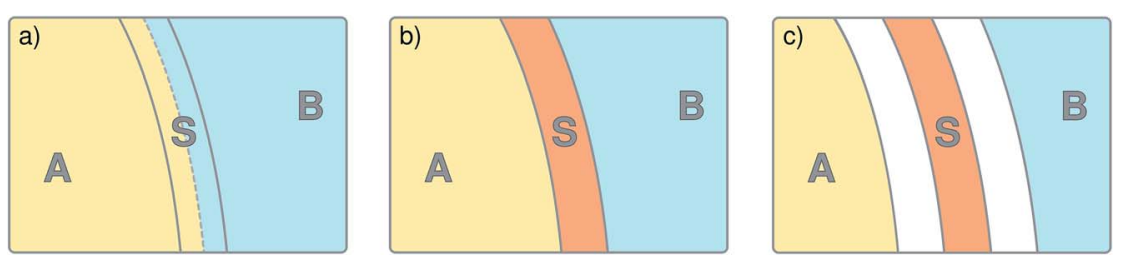

Fig. 1 Schematic representation of the S-shooting approach. In addition to the reactant state, $q(x)<q_{A}$, and the product state, $q(x)>q_{B}$, we introduce a state $S$, defined by $q_{S}^{\min }<$ $q(x)<q_{S}^{\max }$, which is chosen such that any trajectory crossing from A to B or vice versa must cross S. As long as this criterion is obeyed, any arrangement of $A, B$ and $S$ is valid. (a) Regions $A$ and $B$ are separated by a dividing surface (dashed line) and $S$ overlaps with both stable states. Regions defined in this manner are used to obtain cavitation rates in water under tension in Section 3.2. (b) Region $\mathrm{S}$ is located between the stable states $\mathrm{A}$ and $\mathrm{B}$, adjacent to both. (c) Region $S$ is located between the stable states $A$ and $B$, where these regions are separated by areas not corresponding to any of the three states. We employ this setup to compute the reaction rate constant for a Brownian walker in a double-well potential in Section 3.1. 
density $P_{\mathrm{S}}[x(\tau)]$ of trajectories $x(\tau)$ that have at least one configuration in $\mathrm{S}$ is then given by

$$
P_{\mathrm{S}}[x(\tau)]=\frac{P[x(\tau)] H_{\mathrm{S}}[x(\tau)]}{\int \mathscr{D} x(\tau) P[x(\tau)] H_{\mathrm{S}}[x(\tau)]},
$$

where $\int \mathscr{D} x(\tau)$ indicates a summation over all trajectories $x(\tau)$ and the path function $H_{\mathrm{S}}[x(\tau)]$ gives unity if the trajectory $x(\tau)$ has at least one configuration in $\mathrm{S}$ and zero otherwise. The integral in the denominator on the right-hand side of the equation normalizes the distribution. In the above equation, $P[x(\tau)]=\rho\left(x_{0}\right) \prod_{i=0}^{L-1} p\left(x_{i} \rightarrow x_{i+1}\right)$ is the probability of a trajectory $x(\tau)$ in the unconstrained ensemble of trajectories. In writing this expression, we have assumed that the initial conditions $x_{0}$ are distributed according to the equilibrium probability density $\rho\left(x_{0}\right)$, and that the dynamics are Markovian such that the total path probability can be written as the product of short time transition probabilities $p\left(x_{i} \rightarrow x_{i+1}\right)$. In the ensemble of eqn (5), the path indicator function $H_{\mathrm{S}}[x(\tau)]$ assigns a vanishing statistical weight to any trajectory $x(\tau)$ that has no point in $\mathrm{S}$, thereby restricting the S-ensemble to trajectories visiting $\mathrm{S}$.

As shown in detail in Appendix A.1, the time correlation function $C_{\mathrm{AB}}(t)$ can be expressed in terms of path averages in the S-ensemble,

$$
C_{\mathrm{AB}}(t)=\left\langle h_{\mathrm{A}}(0) h_{\mathrm{B}}(t)\right\rangle_{\mathrm{S}} \frac{L+1}{\left\langle N_{\mathrm{S}}[x(\tau)]\right\rangle_{\mathrm{S}}} \frac{\left\langle h_{\mathrm{S}}\right\rangle}{\left\langle h_{\mathrm{A}}\right\rangle} .
$$

Here, $\langle\ldots\rangle_{\mathrm{S}}$ denotes a path average over trajectories $x(\tau)$ in the S-ensemble and $N_{\mathrm{S}}[x(\tau)]$ is the number of configurations of $x(\tau)$ in $\mathrm{S}$ (out of $L+1$ total configurations). The characteristic function $h_{\mathrm{S}}$ for region $\mathrm{S}$ is defined analogously to eqn (1). Conveniently, all quantities appearing in the equation above are either obtained from a free energy computation along $q$, namely $\left\langle h_{\mathrm{A}}\right\rangle$ and $\left\langle h_{\mathrm{S}}\right\rangle$, or from sampling the trajectories touching S. Note that by considering trajectories of length $\tau$ in the S-ensemble, the path average $\left\langle h_{\mathrm{A}}(0) h_{\mathrm{B}}(t)\right\rangle_{\mathrm{S}}$ appearing in eqn (6) can be evaluated for all times $0 \leq t \leq \tau$.

\subsection{Sampling the S-ensemble}

In order to make the equation above useful for the calculation of the time correlation function $C_{\mathrm{AB}}(t)$, and hence for computing the transition rate constant $k_{\mathrm{AB}}$, one needs an efficient way to sample the S-ensemble of trajectories. The ratio $\left\langle h_{\mathrm{S}}\right\rangle /\left\langle h_{\mathrm{A}}\right\rangle$ of equilibrium probabilities needed in eqn (6) can be obtained by free energy calculation methods, e.g., umbrella sampling. By doing so, one also obtains a set of configurations $x_{i} \in \mathrm{S}$ distributed according to their equilibrium probabilities. Each of these configurations can be viewed as a time-slice of a path which has at least one configuration in S, and consequently these configurations can be used as shooting points from which trajectories touching $\mathrm{S}$ are generated. So, let us consider the following algorithm to create trajectories of length $L+1$ that are guaranteed to have at least one configuration in $\mathrm{S}$ :

Step 1. Generate a state $x$ in $\mathrm{S}$ from the equilibrium probability distribution restricted to $\mathrm{S}$, 


$$
\rho_{\mathrm{S}}(x)=\frac{\rho(x) h_{\mathrm{S}}(x)}{\int \mathrm{d} x \rho(x) h_{\mathrm{S}}(x)}
$$

Step 2. Select an integer number $n$ between 0 and $L$ at random. The state $x$ created in the previous step is now considered to be $x_{n}$, that is, the $n$th configuration of the trajectory to be created.

Step 3. Starting from $x_{n}$, perform $L-n$ dynamics steps forward and $n$ steps backward in time to create a trajectory starting at $x_{0}$ which consists of $L+1$ configurations.

The algorithm outlined above can generate all possible trajectories which have at least one configuration in $\mathrm{S}$ and, as such, all trajectories occurring in the desired S-ensemble. However, a closer analysis of the probability to generate a particular trajectory $x(\tau)$ using this procedure indicates that the resulting trajectories are not distributed according to the path probability density $P_{\mathrm{S}}[x(\tau)]$. Rather, they are distributed according to a path probability density $P_{\mathrm{G}}[x(\tau)]$, which gives a larger statistical weight to trajectories with many configurations lying in S. Since a trajectory $x(\tau)$ with $N_{\mathrm{S}}[x(\tau)]$ configurations in $\mathrm{S}$ has $N_{\mathrm{S}}[x(\tau)]$ possible shooting points from which it can be generated and all of them are selected with the same probability, the probability of $x(\tau)$ is proportional to $N_{\mathrm{S}}[x(\tau)]$, i.e., $P_{\mathrm{G}}[x(\tau)]$ $\propto N_{\mathrm{S}}[x(\tau)] P_{\mathrm{S}}[x(\tau)]$. In order to account for the different weights assigned to trajectories by the procedure outlined above, eqn (6) needs to be modified to recover the correlation function $C_{\mathrm{AB}}(t)$ in terms of averages in the ensemble of trajectories produced by the algorithm:

$$
C_{\mathrm{AB}}(t)=(L+1)\left\langle\frac{h_{\mathrm{A}}(0) h_{\mathrm{B}}(t)}{N_{\mathrm{S}}[x(\tau)]}\right\rangle_{\mathrm{G}} \frac{\left\langle h_{\mathrm{S}}\right\rangle}{\left\langle h_{\mathrm{A}}\right\rangle} .
$$

Here, $\langle\ldots\rangle_{\mathrm{G}}$ denotes an average in the ensemble $P_{\mathrm{G}}[x(\tau)]$ generated by the algorithm. A detailed derivation of this result is provided in Appendix A.2.

So far, we have assumed that the starting points $x \in \mathrm{S}$ harvested from free energy calculation methods are distributed according to their Boltzmann weight. However, in many cases these configurations are sampled under the influence of a bias, for instance by employing a parabolic potential in umbrella sampling. By expressing the time correlation function $C_{\mathrm{AB}}(t)$ in terms of path averages over the ensemble $P_{\mathrm{B}}[x(\tau)]$ of trajectories generated by shooting from points obtained using a bias potential, eqn (8) can be easily generalized (see Appendix A.3).

\subsection{Improving sampling by shifting the pathway origins}

The computation of the correlation function $C_{\mathrm{AB}}(t)$ in the ensemble $P_{\mathrm{G}}[x(\tau)]$ can be performed more efficiently by treating long trajectories as a collection of shorter pathways with shifted starting points. Imagine generating one pathway consisting of $2 L+1$ configurations from a starting configuration $x$ in $S$ by propagating $L$ steps in the forward and in the backward direction. From the resulting long trajectory, one can extract a total $L+1$ shorter trajectories of length $L+1$, one starting in $x_{0}$, one in $x_{1}$ and so on up to the trajectory starting at the shooting point $x_{\mathrm{L}}=x$ (see Fig. 2). Each of these trajectories contains at least one point in S, namely the shooting point $x$, and is thus a member of the desired ensemble. Moreover, each of the resulting trajectories is created with the same probability by the algorithm 


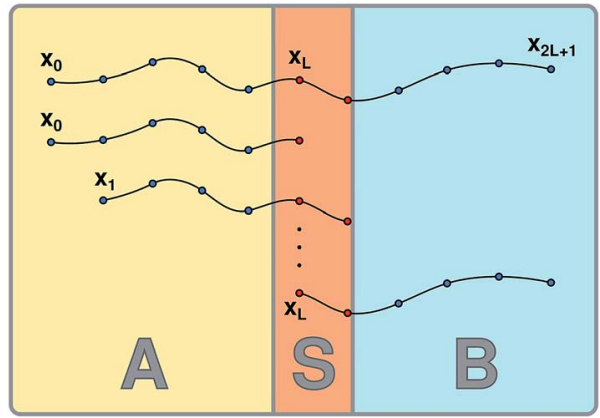

Fig. 2 A long pathway consisting of $2 L+1$ time-slices (top) can be viewed as the envelope of $L+1$ trajectories of length $L$ (below). Note that each of the paths contains at least one configuration in $S$, namely the original shooting point $x_{\mathrm{L}}$, and is thus a member of the S-ensemble.

(see eqn (28) in the Appendix), so one can simply average over these pathways by way of the improved algorithm outlined below:

Step 1. Generate a state $x$ from the probability distribution

$$
\rho_{\mathrm{S}}(x)=\frac{\rho(x) h_{\mathrm{S}}(x)}{\int \mathrm{d} x \rho(x) h_{\mathrm{S}}(x)} .
$$

Step 2. Starting from $x$, perform $L$ dynamics steps forward and $L$ steps backward in time. Here, $\tau=L \Delta t$ is the maximum length for which the correlation function $C_{\mathrm{AB}}(t)$ is to be computed. In practice, $L$ should be chosen to be sufficiently large such that the system commits to either state A or state B when the trajectory is initiated from a point inside $S$.

Step 3. Run over all $L+1$ possible trajectories that still envelop the original shooting point $x_{\mathrm{L}}$ with the initial points $x_{0}, \ldots, x_{\mathrm{L}}$ and compute $N_{\mathrm{S}}[x(\tau)]$ for each trajectory.

Step 4. Update the path average appearing in eqn (8) and repeat.

Once the path average $\left\langle h_{\mathrm{A}}(0) h_{\mathrm{B}}(t) / N_{\mathrm{S}}[x(\tau)]\right\rangle_{\mathrm{G}}$ has been determined according to this method, it can be combined with the ratio $\left\langle h_{\mathrm{S}}\right\rangle /\left\langle h_{\mathrm{A}}\right\rangle$, determined from the free energy calculation, to yield the time correlation function $C_{\mathrm{AB}}(t)$. The transition rate constant $k_{\mathrm{AB}}$ is then obtained from $C_{\mathrm{AB}}(t)$ by numerical differentiation (or fit of a straight line) in its linear regime (provided it exists).

\section{Results and discussion}

\subsection{Brownian walker in a double-well}

We demonstrate the algorithm by applying it to a simple model for an activated process, namely, a one-dimensional Brownian walker in a double-well potential. For this model, the correlation function $\left\langle h_{\mathrm{A}}(0) h_{\mathrm{B}}(t)\right\rangle_{\mathrm{S}}$ can be computed in a direct simulation, providing a point of comparison to the results of biased and unbiased S-shooting. The system is supposed to obey over-damped Langevin dynamics,

$$
x_{t+1}=x_{t}+\beta D F\left(x_{t}\right) \Delta t+\sqrt{2 D \Delta t} \xi,
$$


where $\beta$ is the inverse temperature, $D$ is the diffusion constant, $F(x)=-\mathrm{d} U(x) / \mathrm{d} x$ is the force exerted on the particle by the underlying double-well potential

$$
U(x)=\left(x^{2}-1\right)^{2},
$$

and $\xi$ is delta-correlated Gaussian white noise with zero mean and unit variance. The chosen parameters are $D=1.0, \beta=4.0$ and $\Delta t=0.001$. The regions $\mathrm{A}, \mathrm{S}$ and $\mathrm{B}$ are defined as $x<-0.4,-0.1<x<0.1$ and $0.4<x$, respectively (a schematic representation of the chosen region boundaries is shown in Fig. 1c).

As a reference, we obtain the correlation function $\left\langle h_{\mathrm{A}}(0) h_{\mathrm{B}}(t)\right\rangle_{\mathrm{S}}$ from a single long trajectory produced by a straightforward Brownian dynamics simulation of $5 \times 10^{8}$ time steps. This is done by averaging over all trajectory segments of length $\tau=0.5$ with at least one point in S. Then, we compute the correlation function $\left\langle h_{\mathrm{A}}(0) h_{\mathrm{B}}(t)\right\rangle_{\mathrm{S}}$ by S-shooting, integrating forward and backward in time from points in $\mathrm{S}$ sampled by a Monte Carlo simulation with random displacements drawn from a Gaussian distribution. The whole procedure was repeated by carrying out this Monte Carlo simulation under the influence of a bias $U_{\mathrm{b}}=x^{2} / 2$, thereby obtaining the starting points for biased S-shooting. In terms of averages obtained in the ensemble of trajectories sampled by the algorithm, the correlation function is given by

$$
\left\langle h_{\mathrm{A}}(0) h_{\mathrm{B}}(t)\right\rangle_{\mathrm{S}}=\frac{\left\langle h_{\mathrm{A}}(0) h_{\mathrm{B}}(t) / N_{\mathrm{S}}[x(\tau)]\right\rangle_{\mathrm{G}}}{\left\langle 1 / N_{\mathrm{S}}[x(\tau)]\right\rangle_{\mathrm{G}}}
$$

in the case without bias [see eqn (32)] and by

$$
\left\langle h_{\mathrm{A}}(0) h_{\mathrm{B}}(t)\right\rangle_{\mathrm{S}}=\frac{\left\langle h_{\mathrm{A}}(0) h_{\mathrm{B}}(t) / B[x(\tau)]\right\rangle_{\mathrm{B}}}{\langle 1 / B[x(\tau)]\rangle_{\mathrm{B}}}
$$

in the case with bias [see eqn (40)].

The agreement between the two variants of S-shooting and the straightforward simulation is shown in Fig. 3 and 4. After an initial transient, the correlation function $\left\langle h_{\mathrm{A}}(0) h_{\mathrm{B}}(t)\right\rangle_{\mathrm{S}}$ enters a linear regime. Consequently, its time derivative exhibits a plateau whose value can be used in the estimate for the reaction rate constant $k_{\mathrm{AB}}$. Using the averages $\left\langle h_{\mathrm{S}}\right\rangle=0.00407$ and $\left\langle h_{\mathrm{A}}\right\rangle=0.487$ obtained from

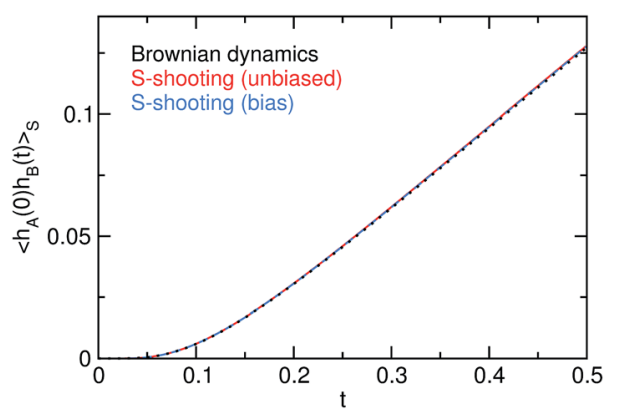

Fig. 3 Correlation function $\left\langle h_{\mathrm{A}}(0) h_{\mathrm{B}}(t)\right\rangle_{\mathrm{S}}$ for a Brownian walker in a double-well potential. The estimates for the correlation function obtained by the S-shooting method (red and blue lines) agree perfectly with the results of the straightforward Brownian dynamics simulation (black line). 


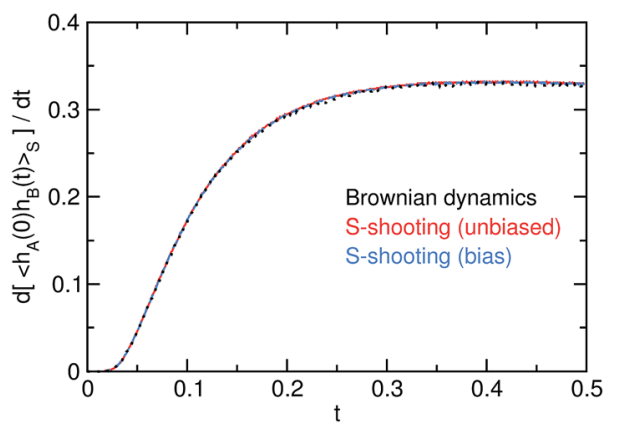

Fig. 4 Numerical time derivative $\mathrm{d}\left\langle h_{\mathrm{A}}(0) h_{\mathrm{B}}(t)\right\rangle_{\mathrm{S}} / \mathrm{d} t$ of the correlation function. Note the plateau emerging after the initial transient behavior at $t \approx 0.3$; this value enters the computation of $k_{\mathrm{AB}}$.

the straightforward simulation as well as the path average $\left\langle N_{\mathrm{S}}[x(\tau)]\right\rangle_{\mathrm{S}}=24.58$, we obtain the rate constant $k_{\mathrm{AB}}=0.056$.

Analysis of the convergence of the rate constants as a function of the number of generated pathways indicates that the statistical error in the rate constants obtained by S-shooting is similar to that of divided saddle theory (provided the same method is used for the free energy calculation). Both methods extract the reaction rate constants from the same set of dynamical trajectories such that this equivalence of their efficiencies is not so surprising. Since divided saddle theory has been shown ${ }^{18}$ to compare well with the reactive flux method of Bennett and Chandler using the effective positive flux approach for the calculation of the transmission coefficient, ${ }^{21}$ this is the case also for the S-shooting method.

\subsection{Cavitation in water under tension}

Liquids can sustain remarkably strong tensions due to the free energetic cost associated with the formation of a liquid-vapor interface which impedes an immediate transition to the vapor phase. Water, in particular, can exist in such a metastable state for a long time before decaying into the vapor phase via cavitation, i.e., bubble nucleation, which has implications for the behavior of various biological systems ${ }^{22-26}$ and for technical applications. ${ }^{27,28}$ As a further demonstration of S-shooting, we now use it to compute the cavitation rate, i.e., the number of cavitation events per unit time and unit volume, of liquid water at different negative pressures (the cavitation free energy and cavitation rates were first obtained in ref. 29). Specifically, we consider a system of $N=2000$ water molecules interacting via the TIP4P/2005 potential ${ }^{30}$ with long range forces treated with Ewald sums. This system is exposed to pressures ranging from $p=$ $-105 \mathrm{MPa}$ to $p=-165 \mathrm{MPa}$ at a temperature of $T=296.4 \mathrm{~K}$, where the equation of state is known at moderate negative pressures from experiments. ${ }^{31}$

To compute the free energy of bubble formation, one must be able to detect bubbles and determine their size for any molecular configuration of this system. This is accomplished using a grid-based procedure that is calibrated to give a thermodynamically consistent estimate for the volume of a bubble. ${ }^{32}$ The bubble volume obtained in this way corresponds to the average increase in system volume due to the presence of a bubble compared to the unconstrained 
metastable liquid at the same conditions. We use the volume of the largest bubble present in the system, $v$, as the reaction coordinate. The free energy, $F(\nu)$, as a function of the bubble volume $v$ is given by $F(\nu)=-\ln \left[\nu_{0} p(\nu)\right]$, where $p(\nu)$ is the probability density of encountering a configuration with a largest bubble of size $v$ and $\nu_{0}$ is an arbitrary constant volume required to make the argument of the logarithm dimensionless. We computed $p(\nu)$, and from it the free energy $F(\nu)$, by employing hybrid Monte Carlo ${ }^{33,34}$ umbrella sampling ${ }^{35}$ with "hard" windows in the isobaric-isothermal ensemble.

Free energy profiles $F(\nu)$ computed for several pressures are shown in Fig. 5. The shape of these curves can be understood in the general framework of classical nucleation theory. The tension applied to the metastable liquid favors the formation and subsequent growth of bubbles through a gain in mechanical work, $p \nu$, by expanding the system under tension. This contribution, in conjunction with the free energy cost of forming the interface ( $A \gamma$, where $A$ is the surface of the bubble and $\gamma$ is the surface tension), leads to a barrier in the free energy which separates the metastable liquid from the vapor (shown in Fig. 5). Once the system overcomes this barrier, it transitions to the vapor phase, which, in contrast to the liquid, cannot sustain tension. Consequently, there is no stable basin on the vapor side of the free energy barrier.

In order to apply the S-shooting formalism to the calculation of bubble nucleation rates, we need to define the stable regions A and $\mathrm{B}$ as well as the transition region $\mathrm{S}$. Based on the computed free energy profiles, we define the region $S$ to be located around the top of the free barrier and regions A and B left and right of the barrier. A schematic representation of the regions A, B and S employed here is shown in Fig. 1a and a detailed list of the region boundaries is given in Table 1. Once the regions are defined, the averages $\left\langle h_{\mathrm{A}}\right\rangle$ and $\left\langle h_{\mathrm{S}}\right\rangle$ needed for the rate calculation can be determined from the free energy profiles. Next, we need to generate dynamical trajectories from region $\mathrm{S}$ in order to compute the correlation function $\left\langle h_{\mathrm{A}}(0) h_{\mathrm{B}}(t)\right\rangle_{\mathrm{S}}$. Starting from configurations in the region $\mathrm{S}$ generated in the free energy calculations, we create pathways by propagating the system backward and forward in time at constant pressure ${ }^{36}$ and temperature ${ }^{37,38}$

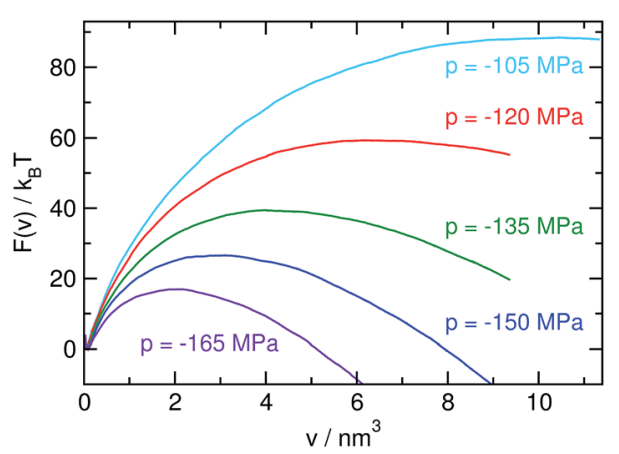

Fig. 5 Free energy $F=-\ln \left[v_{0} p(v)\right]$ as a function of the volume $v$ of the largest bubble, where $v_{0}$ is an arbitrary constant volume, for various pressures. Due to the gain in mechanical work $p v$ associated with the formation of a bubble, the height of the barrier and the size of the critical bubble decrease with increasing tension. Curves are shifted such that the lowest point in the liquid basin aligns for all pressures. 
Table 1 Cavitation rates, J, for various pressures $p$

\begin{tabular}{lllllll}
\hline & & & & $\langle V\rangle^{-1}\left\langle h_{\mathrm{S}}\right\rangle /\left\langle h_{\mathrm{A}}\right\rangle^{a} /$ & \\
$p / \mathrm{MPa}$ & $\mathrm{A} / \mathrm{nm}^{3}$ & $\mathrm{~B} / \mathrm{nm}^{3}$ & $\mathrm{~S} / \mathrm{nm}^{3}$ & $1 /\left\langle N_{\mathrm{S}}\right\rangle_{\mathrm{S}}$ & $\mathrm{nm}^{-3}$ & $J^{b} / \mathrm{nm}^{-3} \mathrm{ps}^{-1}$ \\
-105 & $\nu<10.3$ & $\nu>10.3$ & $10.1<\nu<10.5$ & $2.63 \times 10^{-3}$ & $3.77 \times 10^{-40}$ & $1.98 \times 10^{-41}$ \\
-120 & $\nu<6.25$ & $\nu>6.25$ & $6.05<\nu<6.45$ & $2.40 \times 10^{-3}$ & $2.54 \times 10^{-27}$ & $1.05 \times 10^{-28}$ \\
-135 & $\nu<4.075$ & $\nu>4.075$ & $3.85<\nu<4.3$ & $2.03 \times 10^{-3}$ & $8.92 \times 10^{-19}$ & $2.81 \times 10^{-20}$ \\
-150 & $\nu<3.06$ & $\nu>3.06$ & $2.95<\nu<3.17$ & $3.42 \times 10^{-3}$ & $1.30 \times 10^{-13}$ & $6.81 \times 10^{-15}$ \\
-165 & $\nu<2.095$ & $\nu>2.095$ & $1.985<\nu<2.205$ & $2.89 \times 10^{-3}$ & $2.19 \times 10^{-9}$ & $9.75 \times 10^{-11}$ \\
The equilibrium probability ratio $\left\langle h_{\mathrm{S}}\right\rangle /\left\langle h_{\mathrm{A}}\right\rangle$ was obtained from the free energy data shown \\
Th Fig. 5 and $\langle V\rangle$ is the average volume of the metastable liquid at the appropriate pressure. \\
The cavitation rate $J$ is obtained by combining the equilibrium probability ratio with a fit to \\
The long-time tail of the time derivative of the correlation function from Fig. 7.
\end{tabular}

using a time-reversible integrator. ${ }^{39-41}$ In order to keep the computational cost manageable, the trajectories used in the rate computation are propagated until they reach a fixed value along the reaction coordinate, rather than for a fixed time. These fixed values were chosen such that they correspond to being approximately $10 k_{\mathrm{B}} T$ lower than the top of the free energy barrier, at which point trajectories are unlikely to re-cross the barrier. $\dagger$ Since this approach leads to trajectories of varying length, trajectories shorter than the desired trajectory length $2 L+1$ were padded with their final value on either side of the barrier when computing the correlation function $C_{\mathrm{AB}}(t)$.

Correlation functions $\left\langle h_{\mathrm{A}}(0) h_{\mathrm{B}}(t)\right\rangle_{\mathrm{s}}$, obtained via eqn (32) from trajectories in the ensemble $P_{\mathrm{G}}[x(\tau)]$, are shown in Fig. 6 . Since the state $\mathrm{S}$ fully overlaps with the adjacent states $\mathrm{A}$ and $\mathrm{B}$, one encounters non-finite contributions to the correlation $\left\langle h_{\mathrm{A}}(0) h_{\mathrm{B}}(t)\right\rangle_{\mathrm{S}}$ even for short times, leading to a steep slope for small $t$ (in contrast to the shape of $\left\langle h_{\mathrm{A}}(0) h_{\mathrm{B}}(t)\right\rangle_{\mathrm{S}}$ observed in Section 3.1). Its time derivative,

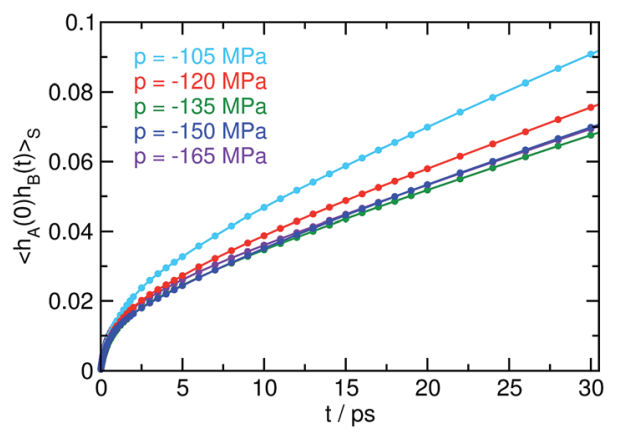

Fig. 6 Correlation function $\left\langle h_{\mathrm{A}}(0) h_{\mathrm{B}}(t)\right\rangle_{\mathrm{S}}$. Note the difference in shape compared to Fig. 3 due to the different boundaries of the states $A$ and $B$.

\footnotetext{
$\dagger$ For the two lowest tensions investigated, $p=-105 \mathrm{MPa}$ and $p=-120 \mathrm{MPa}$, we extrapolated the free energy to higher bubble volumes to determine the limiting value of the order parameter.
} 


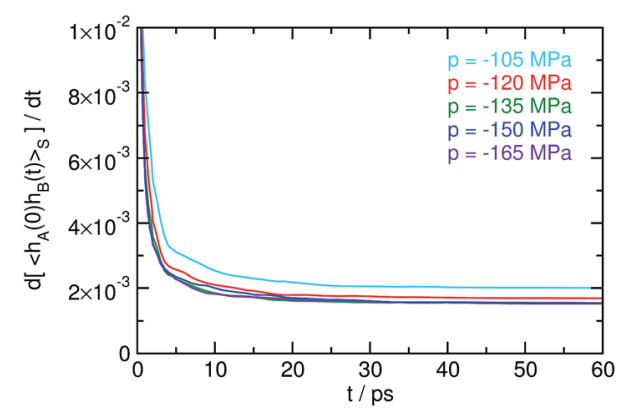

Fig. 7 Time derivative $\mathrm{d}\left\langle h_{\mathrm{A}}(0) h_{\mathrm{B}}(t)\right\rangle_{\mathrm{S}} / \mathrm{d} t$ of the correlation function depicted in Fig. 6 . A fit to the emerging plateau for long times is used in the computation of cavitation rates.

shown in Fig. 7, exhibits transient behavior followed by the emergence of a plateau for longer times. The resulting cavitation rates $J=k_{\mathrm{AB}} /\langle V\rangle$, which span almost 40 orders of magnitude over the investigated range of pressures, are presented in Table 1.

\section{Conclusion}

In the study of rare transitions between long-lived stable states, one often uses large numbers of short molecular dynamics trajectories to determine committors or estimate diffusion coefficients along some collective variable of interest. Here we have presented an algorithm to calculate reaction rate constants by combining dynamical information extracted from such brief trajectories with the results of free energy calculations. For the method to be computationally efficient, the trajectories need to be initiated close to the transition state region such that they have a non-negligible probability to connect the stable states. Hence, the method follows the central idea of the Bennett-Chandler approach, ${ }^{19,20}$ in which one first computes the transition state theory approximation of the reaction rate constant based on the free energy, and then applies a dynamical correction obtained from short trajectories started on a dividing surface separating the stable states. Our approach uses the same information as the divided saddle theory of Daru and Stirling, ${ }^{18}$ but processes it in a different way to yield the time correlation function of the stable state populations, from which the reaction rate constant is obtained by taking a time derivative. Knowledge of the correlation function also yields information about the barrier crossing dynamics and permits to verify whether the kinetics follows the exponential behavior expected from the phenomenological rate equations.

Just like the Bennett-Chandler approach and divided saddle theory, the new method, which is equally applicable to under- and overdamped dynamics, also requires a priori knowledge of a reaction coordinate. The reaction coordinate needs to provide an at least rough measure for the progress of the transition and it can be either continuous or discrete, such as the size of the largest crystalline cluster usually used in crystallization studies. As an illustration, we have applied the procedure to a Brownian walker in a double well potential and to cavitation in 
water at negative pressures, demonstrating that it can be used to determine reaction rate constants in complex condensed environments.

\section{A Derivation of the time correlation function in the S-shooting formalism}

\section{A.1 The time correlation function in the S-ensemble}

The time correlation function

$$
C_{\mathrm{AB}}(t)=\frac{\left\langle h_{\mathrm{A}}(0) h_{\mathrm{B}}(t)\right\rangle}{\left\langle h_{\mathrm{A}}\right\rangle},
$$

which equals the conditional probability to find the system in B at time $t$ provided it was in $\mathrm{A}$ at time 0 , can be written as an average in the ensemble $P[x(\tau)]$ of trajectories $x(\tau)$ of length $\tau \geq t$ :

$$
C_{\mathrm{AB}}(t)=\frac{\int \mathscr{D} x(\tau) P[x(\tau)] h_{\mathrm{A}}\left(x_{0}\right) h_{\mathrm{B}}\left(x_{t}\right)}{\int \mathrm{d} x_{0} \rho\left(x_{0}\right) h_{\mathrm{A}}\left(x_{0}\right)},
$$

where the notation $\int \mathscr{D} x(\tau)$ indicates a summation over all pathways $x(\tau), x_{t}$ is the microscopic state (which we also call configuration or point) of the system at time $t$, and the probability density of a trajectory consisting of $L+1$ configurations is given by

$$
P[x(\tau)]=\rho\left(x_{0}\right) \prod_{i=0}^{L-1} p\left(x_{i} \rightarrow x_{i+1}\right) .
$$

Here, $\rho\left(x_{0}\right)$ is the equilibrium probability density of the configuration $x_{0}$ in the thermodynamic ensemble of interest and $p\left(x_{i} \rightarrow x_{i+1}\right)$ is the probability density of reaching configuration $x_{i+1}$ when the system in configuration $x_{i}$ is propagated by one step. The time correlation function $C_{\mathrm{AB}}(t)$ contains all the information needed to determine the rate constant $k_{\mathrm{AB}}$ for transitions from $\mathrm{A}$ to $\mathrm{B}$, which is equal to the time derivative of $C_{\mathrm{AB}}(t)$ in its linear regime. If transitions from A to B are rare, it is difficult to determine $C_{\mathrm{AB}}(t)$ from straightforward molecular dynamics simulations. In the following we present an algorithm to determine $C_{\mathrm{AB}}(t)$ that is not affected by this limitation.

Although the integral in eqn (15) extends over all possible trajectories, the nonvanishing contributions to the correlation function $C_{\mathrm{AB}}(t)$ stem from trajectories that start in A and reach the product state B by the time $t$. As such, one can obtain the correlation function $C_{\mathrm{AB}}(t)$ by sampling from a constrained ensemble, provided that the constrained ensemble contains all trajectories going from A to B. To define such an ensemble, we introduce the additional region $\mathrm{S}$ located such that any trajectory transitioning from A to B necessarily crosses S, i.e., has at least one point in S (see Fig. 1). The correlation function can then be written as

$$
C_{\mathrm{AB}}(t)=\frac{\left\langle h_{\mathrm{A}}(0) h_{\mathrm{B}}(t)\right\rangle}{\left\langle h_{\mathrm{A}}\right\rangle}=\frac{\left\langle h_{\mathrm{A}}(0) h_{\mathrm{B}}(t) H_{\mathrm{S}}[x(\tau)]\right\rangle}{\left\langle h_{\mathrm{A}}\right\rangle} .
$$

Here, the path function $H_{\mathrm{S}}[x(\tau)]$ is unity if the trajectory $x(\tau)$ has at least one point in $S$ and zero otherwise. We can insert $H_{\mathrm{S}}[x(\tau)]=1$ in the average above 
without changing the correlation function, because if both $h_{\mathrm{A}}(0)=1$ and $h_{\mathrm{B}}(t)=1$ then $H_{\mathrm{S}}[x(\tau)]=1$, and if $h_{\mathrm{A}}(0)=0$ or $h_{\mathrm{B}}(t)=0$ the value of $H_{\mathrm{S}}[x(\tau)]$ does not matter. Multiplying and dividing by $\left\langle H_{\mathrm{S}}[x(\tau)]\right\rangle$ one obtains

$$
C_{\mathrm{AB}}(t)=\frac{\left\langle h_{\mathrm{A}}(0) h_{\mathrm{B}}(t) H_{\mathrm{S}}[x(\tau)]\right\rangle}{\left\langle H_{\mathrm{S}}[x(\tau)]\right\rangle} \frac{\left\langle H_{\mathrm{S}}[x(\tau)]\right\rangle}{\left\langle h_{\mathrm{A}}\right\rangle}=\left\langle h_{\mathrm{A}}(0) h_{\mathrm{B}}(t)\right\rangle_{\mathrm{S}} \frac{\left\langle H_{\mathrm{S}}[x(\tau)]\right\rangle}{\left\langle h_{A}\right\rangle} .
$$

Here, $\langle R\rangle_{\mathrm{S}}=\left\langle R[x(\tau)] H_{\mathrm{S}}[x(\tau)]\right\rangle /\left\langle H_{\mathrm{S}}[x(\tau)]\right\rangle$ is the average for an arbitrary path property $R[x(\tau)]$ in the ensemble $P_{\mathrm{S}}[x(\tau)]$ of paths with at least one point in the region $\mathrm{S}$,

$$
P_{\mathrm{S}}[x(\tau)]=\frac{P[x(\tau)] H_{\mathrm{S}}[x(\tau)]}{\int \mathscr{D} x(\tau) P[x(\tau)] H_{\mathrm{S}}[x(\tau)]},
$$

where the denominator normalizes the distribution. Since any reactive trajectory, i.e., any trajectory connecting $\mathrm{A}$ and $\mathrm{B}$, has to have points in $\mathrm{S}$, the ensemble $P_{\mathrm{S}}[x(\tau)]$ contains all reactive trajectories, albeit with a statistical weight that differs from that in the equilibrium trajectory ensemble $P[x(\tau)]$ by the factor $\left\langle H_{\mathrm{S}}[x(\tau)]\right\rangle$, the probability of an equilibrium trajectory of length $\tau$ to visit $\mathrm{S}$.

In order to evaluate $C_{\mathrm{AB}}(t)$ according to eqn (18) using trajectories sampled from $P_{\mathrm{S}}[x(\tau)]$, we write eqn (18) as

$$
C_{\mathrm{AB}}(t)=\left\langle h_{\mathrm{A}}(0) h_{\mathrm{B}}(t)\right\rangle_{\mathrm{S}} \frac{\left\langle H_{\mathrm{S}}[x(\tau)]\right\rangle}{\left\langle h_{\mathrm{S}}\right\rangle} \frac{\left\langle h_{\mathrm{S}}\right\rangle}{\left\langle h_{\mathrm{A}}\right\rangle},
$$

where $\left\langle h_{\mathrm{S}}\right\rangle=\int_{q_{\mathrm{S}}^{\min }}^{q_{\mathrm{S}}^{\max }} p(q) \mathrm{d} q$ is the equilibrium population of the region $\mathrm{S}$, such that the ratio $\left\langle h_{\mathrm{S}}\right\rangle /\left\langle h_{\mathrm{A}}\right\rangle$ can be obtained from the free energy $F(q)=-k_{\mathrm{B}} T \ln p(q)$ computed as a function of the reaction coordinate $q$. Since $\left\langle h_{\mathrm{A}}(0) h_{\mathrm{B}}(t)\right\rangle_{\mathrm{S}}$ can be determined as a path average over the ensemble $P_{\mathrm{S}}[x(\tau)]$, all that is still needed to compute $C_{\mathrm{AB}}(t)$ is the ratio $\left\langle H_{\mathrm{S}}[x(\tau)]\right\rangle /\left\langle h_{\mathrm{S}}\right\rangle$. Since the dynamics is microscopically reversible and thus fulfills detailed balance, one can express the average $\left\langle h_{\mathrm{S}}\right\rangle$ as a path average,

$$
\left\langle h_{\mathrm{S}}\right\rangle=\int \mathrm{d} x_{i} \rho\left(x_{i}\right) h_{\mathrm{S}}\left(x_{i}\right)=\int \mathscr{D} x(\tau) \rho\left(x_{0}\right) \prod_{j=0}^{L-1} p\left(x_{j} \rightarrow x_{j+1}\right) h_{\mathrm{S}}\left(x_{i}\right),
$$

evaluated at an arbitrary point $x_{i}$ along the trajectories. Since if $h_{\mathrm{S}}\left(x_{i}\right)=1$ for at least one point of the trajectory $x(\tau)$ also $H_{\mathrm{S}}[x(\tau)]=1$, we can insert $H_{\mathrm{S}}[x(\tau)]$ into the average $\left\langle h_{\mathrm{S}}\left(x_{i}\right)\right\rangle$ without changing its value and we obtain

$$
\frac{\left\langle H_{\mathrm{S}}[x(\tau)]\right\rangle}{\left\langle h_{\mathrm{S}}\right\rangle}=\frac{\left\langle H_{\mathrm{S}}[x(\tau)]\right\rangle}{\left\langle h_{\mathrm{S}} H_{\mathrm{S}}[x(\tau)]\right\rangle}=\frac{1}{\left\langle h_{\mathrm{S}}\right\rangle_{\mathrm{S}}} .
$$

But since all configurations $x_{i}$ along a path occur with the same likelihood (see eqn (21)), one can simply average $\left\langle h_{\mathrm{S}}\left(x_{i}\right)\right\rangle_{\mathrm{S}}$ over all $L+1$ time slices:

$$
\left\langle h_{\mathrm{S}}\right\rangle_{\mathrm{S}}=\frac{1}{L+1} \sum_{i=0}^{L}\left\langle h_{\mathrm{S}}\left(x_{i}\right)\right\rangle_{\mathrm{S}}=\frac{1}{L+1}\left\langle N_{\mathrm{S}}[x(\tau)]\right\rangle_{\mathrm{S}}
$$


where $N_{\mathrm{S}}[x(\tau)]=\sum_{i=0}^{L} h_{\mathrm{S}}\left(x_{i}\right)$ is the number of configurations in S. Consequently, $\left\langle h_{\mathrm{S}}\right\rangle_{\mathrm{S}}$ is the fraction of configurations $x_{i}$ of a given path $x(\tau)$ located in the region S. Inserting this result into eqn (20) yields the time-correlation function $C_{\mathrm{AB}}(t)$ expressed in terms of path averages obtained in the ensemble of trajectories visiting $S$,

$$
C_{\mathrm{AB}}(t)=\left\langle h_{\mathrm{A}}(0) h_{\mathrm{B}}(t)\right\rangle_{\mathrm{S}} \frac{L+1}{\left\langle N_{\mathrm{S}}[x(\tau)]\right\rangle_{\mathrm{S}}} \frac{\left\langle h_{\mathrm{S}}\right\rangle}{\left\langle h_{\mathrm{A}}\right\rangle}
$$

\section{A.2 Relation to the ensemble generated by S-shooting}

The algorithm presented in the main text generates trajectories by picking shooting points $x$ in S according to the probability density

$$
\rho_{\mathrm{S}}(x)=\frac{\rho(x) h_{\mathrm{S}}(x)}{\int \mathrm{d} x \rho(x) h_{\mathrm{S}}(x)}=\frac{\rho\left(x_{i}\right) h_{\mathrm{S}}\left(x_{i}\right)}{\left\langle h_{\mathrm{S}}\right\rangle}
$$

and subsequently propagating these configurations forward and backward in time. But which ensemble is generated by this algorithm? Since every trajectory $x(\tau)$ visiting $\mathrm{S}$, and as such all reactive trajectories, has at least one configuration in $\mathrm{S}$ by definition, it can be generated by shooting from points in S. However, in order to verify whether the algorithm generates the desired ensemble, one has to determine how these trajectories are weighted with respect to one another, i.e., one has to inspect the likelihood with which the algorithm generates a particular trajectory. In particular, in doing that one has to take into account that trajectories with multiple points in $\mathrm{S}$ have more than one way to be generated by the algorithm.

Consider a trajectory $x(\tau)=\left\{x_{0}, \cdots, x_{i_{1}}, \cdots, x_{i_{N_{\mathrm{S}}[x(\tau)]}}, \cdots, x_{\mathrm{L}}\right\}$ with $\quad N_{\mathrm{S}}[x(\tau)]$ configurations in region $\mathrm{S}$. (Two examples of such trajectories are shown in Fig. 8.) Here, the $N_{\mathrm{S}}[x(\tau)]$ subscripts $i_{1}, i_{2}, \ldots, i_{\mathrm{N}_{\mathrm{S}}[x(\tau)]}$ are the indices of the configurations of the trajectory $x(\tau)$ that are in S. In the shooting algorithm presented in the main text, the trajectory $x(\tau)$ can be generated by shooting from each of these $N_{\mathrm{S}}[x(\tau)]$ points. Let us now consider the probability density that the trajectory $x(\tau)$ is generated from the first one of these points, $x_{i_{1}}$. For this to happen, configuration $x_{i_{1}}$ must first be selected from $\rho_{\mathrm{S}}\left(x_{i_{1}}\right)$ and then designated to be the $i_{1}$-th configuration along the trajectory by drawing the index $i_{1}$ with probability $1 /(L+1)$ from the integers 0 to $L$. Starting from $x_{i_{1}}$, one then propagates the dynamics for $L-i_{1}$ steps in the forward and $i_{1}$ steps in the backward direction using the rules of the underlying dynamics, such that the probability density $p\left[x(\tau) ; x_{i_{1}}\right]$ to generate exactly trajectory $x(\tau)$ from configuration $x_{i_{1}}$ is given by

$$
p\left[x(\tau) ; x_{i_{1}}\right]=\frac{\rho_{\mathrm{S}}\left(x_{i_{1}}\right)}{L+1} \prod_{i=0}^{i_{1}-1} p\left(\bar{x}_{i+1} \rightarrow \bar{x}_{i}\right) \prod_{i=i_{1}}^{L-1} p\left(x_{i} \rightarrow x_{i+1}\right),
$$

where $\bar{x}$ is the configuration $x$ with reversed momenta (because the first product corresponds to propagating the system backward in time). Since the dynamics is microscopically reversible, the transition probability obeys detailed balance; in particular, 


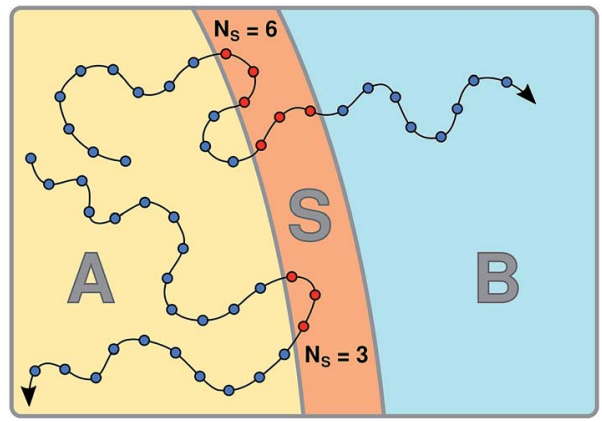

Fig. 8 When shooting points are picked from the region $\mathrm{S}$ according to the probability density $\rho_{\mathrm{S}}(x)$, pathways with a larger number $N_{S}[x(\tau)]$ of configurations in $S$ are more likely to be created. This overcounting of pathways must be corrected for when computing path averages.

$$
\rho\left(x_{i+1}\right) p\left(\bar{x}_{i+1} \rightarrow \bar{x}_{i}\right)=\rho\left(x_{i}\right) p\left(x_{i} \rightarrow x_{i+1}\right),
$$

where we took advantage of the fact that the Hamiltonian does not change when the momenta of a configuration are reversed, i.e., $\rho(\bar{x})=\rho(x)$. Applying eqn (27) to eqn (26) repeatedly, the equilibrium probability density $\rho\left(x_{i_{1}}\right)$, initially applied to $x_{i_{1}}$, is shifted to the first configuration $x_{0}$ of the trajectory:

$$
p\left[x(\tau) ; x_{i_{1}}\right]=\frac{h_{\mathrm{S}}\left(x_{i_{1}}\right)}{(L+1)\left\langle h_{\mathrm{S}}\right\rangle} \rho\left(x_{0}\right) \prod_{i=0}^{L-1} p\left(x_{i} \rightarrow x_{i+1}\right)=\frac{1}{(L+1)\left\langle h_{\mathrm{S}}\right\rangle} P[x(\tau)],
$$

where $h_{\mathrm{S}}\left(x_{i_{1}}\right)=1$ since the shooting point $x_{i_{1}}$ is in $\mathrm{S}$ by construction. The equation above shows that the likelihood of generating $x(\tau)$ is independent of the chosen shooting point and the probability of generating the trajectory from configuration $x_{i_{1}}$ is the same as that of generating it from any of the other possible shooting points $x_{i_{2}}$ to $x_{i_{N_{\mathrm{S}}[(\tau)]}}$. Hence, the total likelihood to generate a particular trajectory $x(\tau)$ by shooting from points in $\mathrm{S}$ is just $N_{\mathrm{S}}[x(\tau)]$ times the likelihood of generating it by shooting from one specific point in $\mathrm{S}$ :

$$
P_{\mathrm{G}}[x(\tau)]=\frac{N_{\mathrm{S}}[x(\tau)] P[x(\tau)] H_{\mathrm{S}}[x(\tau)]}{(L+1)\left\langle h_{\mathrm{S}}\right\rangle},
$$

where we inserted $H_{\mathrm{S}}[x(\tau)]$ in the first line to emphasize that this algorithm only creates trajectories with at least one configuration in $\mathrm{S}$.

The ensemble $P_{\mathrm{G}}[x(\tau)]$ can be expressed in terms of the ensemble $P_{\mathrm{S}}[x(\tau)]$ of trajectories visiting $\mathrm{S}$,

$$
\begin{aligned}
P_{\mathrm{G}}[x(\tau)] & =N_{\mathrm{S}}[x(\tau)] \frac{P[x(\tau)] H_{\mathrm{S}}[x(\tau)]}{\int \mathscr{D} x(\tau) P[x(\tau)] H_{\mathrm{S}}[x(\tau)]} \frac{\int \mathscr{D} x(\tau) P[x(\tau)] H_{\mathrm{S}}[x(\tau)]}{(L+1)\left\langle h_{\mathrm{S}}\right\rangle} \\
& =N_{\mathrm{S}}[x(\tau)] P_{\mathrm{S}}[x(\tau)] \frac{\left\langle H_{\mathrm{S}}[x(\tau)]\right\rangle}{(L+1)\left\langle h_{\mathrm{S}}\right\rangle} .
\end{aligned}
$$

Thus, the likelihood to generate a particular trajectory $x(\tau)$ in the ensemble $P_{\mathrm{G}}[x(\tau)]$ generated by the shooting algorithm is related to the likelihood in the ensemble $P_{\mathrm{S}}[x(\tau)]$ of trajectories visiting $\mathrm{S}$ via $P_{\mathrm{G}}[x(\tau)] \propto N_{\mathrm{S}}[x(\tau)] P_{\mathrm{S}}[x(\tau)]$, i.e., in the 
ensemble $P_{\mathrm{G}}[x(\tau)]$, pathways with multiple points in $\mathrm{S}$ have a likelihood that is too high compared to the ensemble $P_{\mathrm{S}}[x(\tau)]$.

Since $P_{\mathrm{G}}[x(\tau)]$ is normalized [see eqn (22) and (23)], we can now express path averages in the desired ensemble in terms of $P_{\mathrm{G}}[x(\tau)]$. The average $\langle R[x(\tau)]\rangle_{\mathrm{S}}$ of an arbitrary path property $R[x(\tau)]$ is given by

$$
\langle R[x(\tau)]\rangle_{\mathrm{S}}=\frac{\int \mathscr{D} x(\tau) R[x(\tau)] P_{\mathrm{S}}[x(\tau)]}{\int \mathscr{D} x(\tau) P_{\mathrm{S}}[x(\tau)]}
$$

which, by inserting eqn (30), can be re-written as

$$
\langle R[x(\tau)]\rangle_{\mathrm{S}}=\frac{\int \mathscr{D} x(\tau)\left(R[x(\tau)] / N_{\mathrm{S}}[x(\tau)]\right) P_{\mathrm{G}}[x(\tau)]}{\int \mathscr{D} x(\tau)\left(1 / N_{\mathrm{S}}[x(\tau)]\right) P_{\mathrm{G}}[x(\tau)]}=\frac{\left\langle R[x(\tau)] / N_{\mathrm{S}}[x(\tau)]\right\rangle_{\mathrm{G}}}{\left\langle 1 / N_{\mathrm{S}}[x(\tau)]\right\rangle_{\mathrm{G}}} .
$$

Using this expression for averages in the ensemble of pathways visiting $S$ we can rewrite the expression for $C_{\mathrm{AB}}(t)$ in eqn (6) in terms of averages in the ensemble $P_{\mathrm{G}}[x(\tau)]$ :

$$
\begin{aligned}
C_{\mathrm{AB}}(t) & =\left\langle h_{\mathrm{A}}(0) h_{\mathrm{B}}(t)\right\rangle_{\mathrm{S}} \frac{L+1}{\left\langle N_{\mathrm{S}}[x(\tau)]\right\rangle_{\mathrm{S}}} \frac{\left\langle h_{\mathrm{S}}\right\rangle}{\left\langle h_{\mathrm{A}}\right\rangle} \\
& =(L+1) \frac{\left\langle h_{\mathrm{A}}(0) h_{\mathrm{B}}(t) / N_{\mathrm{S}}[x(\tau)]\right\rangle_{\mathrm{G}}}{\left\langle 1 / N_{\mathrm{S}}[x(\tau)]\right\rangle_{\mathrm{G}}} \frac{\left\langle 1 / N_{\mathrm{S}}[x(\tau)]\right\rangle_{\mathrm{G}}}{\left\langle N_{\mathrm{S}}[x(\tau)] / N_{\mathrm{S}}[x(\tau)]\right\rangle_{\mathrm{G}}} \frac{\left\langle h_{\mathrm{S}}\right\rangle}{\left\langle h_{\mathrm{A}}\right\rangle} \\
& =(L+1)\left\langle\frac{h_{\mathrm{A}}(0) h_{\mathrm{B}}(t)}{N_{\mathrm{S}}[x(\tau)]}\right\rangle_{\mathrm{G}} \frac{\left\langle h_{\mathrm{S}}\right\rangle}{\left\langle h_{\mathrm{A}}\right\rangle},
\end{aligned}
$$

thus obtaining eqn (8).

\section{A.3 Biased sampling of shooting points}

The computation of the correlation function $C_{\mathrm{AB}}(t)$ via eqn (6) assumes that the starting points of the trajectories are distributed in $S$ according to their Boltzmann weight. Below, we describe how eqn (6) has to be adapted when the initial points are sampled from a biased distribution instead, that is, the shooting points $x$ are generated according to

$$
\rho_{\mathrm{B}}(x)=\rho_{\mathrm{S}}(x) b(x),
$$

where the weight $b(x)$ assigned to a configuration due to the bias potential $U_{\mathrm{b}}(x)$ is given by

$$
b(x)=\frac{\mathrm{e}^{-\beta U_{\mathrm{b}}(x)}}{\int \mathrm{d} x \rho_{\mathrm{S}}(x) \mathrm{e}^{-\beta U_{\mathrm{b}}(x)}} .
$$

The generation probability $p\left[x(\tau) ; x_{i j}\right]$ for a path $x(\tau)$ by shooting from a specific point $x_{i_{j}}$ in $\mathrm{S}$ is then given by [see eqn (28)]

$$
p\left[x(\tau) ; x_{i_{j}}\right]=\frac{1}{(L+1)\left\langle h_{\mathrm{S}}\right\rangle} P[x(\tau)] b\left(x_{i_{j}}\right) .
$$

As in the previous section, the subscripts $i_{1}$ to $i_{N_{\mathrm{S}}[x(\tau)]}$ are the indices of the $N_{\mathrm{S}}[x(\tau)]$ configurations of the trajectory $x(\tau)$ that are in S. Since the path $x(\tau)$ can be 
generated from any configuration in $\mathrm{S}$, the total generation probability of a path with $N_{\mathrm{S}}[x(\tau)]$ points in $\mathrm{S}$ is

$$
P_{\mathrm{B}}[x(\tau)]=\frac{B[x(\tau)]}{(L+1)\left\langle h_{\mathrm{S}}\right\rangle} P[x(\tau)],
$$

where $B[x(\tau)]=\sum_{j=1}^{N_{\mathrm{S}}[x(\tau)]} b\left(x_{i_{j}}\right)$ and the sum runs over all points in S. Since the trajectories are generated by shooting from points located in $\mathrm{S}$, each of the resulting pathways has at least one configuration in $\mathrm{S}$, which allows us to rewrite the equation above as

$$
P_{\mathrm{B}}[x(\tau)]=\frac{B[x(\tau)]}{(L+1)\left\langle h_{\mathrm{S}}\right\rangle} P[x(\tau)] H_{\mathrm{S}}[x(\tau)]
$$

and, by inserting eqn (22) and (23), one obtains

$$
P_{\mathrm{B}}[x(\tau)]=\frac{B[x(\tau)]}{\left\langle N_{\mathrm{S}}[x(\tau)]\right\rangle_{\mathrm{S}}} \frac{P[x(\tau)] H_{\mathrm{S}}[x(\tau)]}{\left\langle H_{\mathrm{S}}[x(\tau)]\right\rangle}=\frac{B[x(\tau)]}{\left\langle N_{\mathrm{S}}[x(\tau)]\right\rangle_{\mathrm{S}}} P_{\mathrm{S}}[x(\tau)]
$$

Since we aim to formulate the correlation function $C_{\mathrm{AB}}(t)$ in terms of path averages in the ensemble $P_{\mathrm{S}}[x(\tau)]$, we now express the path average $\langle R[x(\tau)]\rangle_{\mathrm{S}}$ in terms of averages calculated in the ensemble $P_{\mathrm{B}}[x(\tau)]$,

$$
\begin{aligned}
\langle R[x(\tau)]\rangle_{\mathrm{S}}= & \int \mathscr{D} x(\tau) R[x(\tau)] P_{\mathrm{S}}[x(\tau)]=\frac{\int \mathscr{D} x(\tau) R[x(\tau)] P_{\mathrm{B}}[x(\tau)]\left\langle N_{\mathrm{S}}[x(\tau)]\right\rangle_{\mathrm{S}} / B[x(\tau)]}{\int \mathscr{D} x(\tau) P_{\mathrm{B}}[x(\tau)]\left\langle N_{\mathrm{S}}[x(\tau)]\right\rangle_{\mathrm{S}} / B[x(\tau)]} \\
= & \frac{\left\langle\frac{R[x(\tau)]}{B[x(\tau)]}\right\rangle_{\mathrm{B}}}{\left\langle\frac{1}{B[x(\tau)]}\right\rangle_{\mathrm{B}}}
\end{aligned}
$$

where we divided by $\int \mathscr{D} x[\tau] P_{\mathrm{S}}[x(\tau)]=1$ in the second line. Note that since the bias sum $B[x(\tau)]$ appears both in the numerator and the denominator, the above expression does not change when the bias is not normalized, i.e., when $\tilde{B}[x(\tau)]=\sum_{j=1}^{N_{\mathrm{S}}[x(\tau)]} \exp \left[-\beta U_{\mathrm{b}}\left(x_{i_{j}}\right)\right]$ is used for convenience.

Using eqn (40) to rewrite eqn (6), we obtain $C_{\mathrm{AB}}(t)$ in terms of biased averages:

$$
C_{\mathrm{AB}}(t)=\left\langle h_{\mathrm{A}}(0) h_{\mathrm{B}}(t)\right\rangle_{\mathrm{S}} \frac{L+1}{\left\langle N_{\mathrm{S}}[x(\tau)]\right\rangle_{\mathrm{S}}} \frac{\left\langle h_{\mathrm{S}}\right\rangle}{\left\langle h_{\mathrm{A}}\right\rangle}=(L+1) \frac{\left\langle\frac{h_{\mathrm{A}}(0) h_{\mathrm{B}}(t)}{\tilde{B}[x(\tau)]}\right\rangle_{\mathrm{B}}}{\left\langle\frac{N_{\mathrm{S}}[x(\tau)]}{\tilde{B}[x(\tau)]}\right\rangle_{\mathrm{B}}} \frac{\left\langle h_{\mathrm{S}}\right\rangle}{\left\langle h_{\mathrm{A}}\right\rangle} .
$$

When shooting points are obtained from an unbiased distribution, then $\tilde{B}[x(\tau)]=N_{\mathrm{S}}[x(\tau)]$ and eqn (8) is recovered.

\section{Acknowledgements}

We thank J. Daru, P. Geiger, and C. Moritz for insightful comments. This work was supported by the Austrian Science Fund (FWF) within the SFB ViCoM [Grant 
F41] as well as under grant P24681-N20. A. S. also acknowledges support from the Vienna Scientific Cluster (VSC) School. Calculations were carried out on the Vienna Scientific Cluster (VSC).

\section{References}

1 P. Hänggi, P. Talkner and M. Borkovec, Rev. Mod. Phys., 1990, 62, 251.

2 D. Chandler, in Barrier Crossings: Classical Theory of Rare but Important Events, ed. B. J. Berne, G. Ciccotti and D. F. Coker, Singapore World Sci., 1998, ch. 1, pp. 3-23.

3 A. M. Berezhkovskii and A. Szabo, J. Phys. Chem. B, 2013, 117, 13115-13119.

4 R. B. Best and G. Hummer, Phys. Rev. Lett., 2006, 96, 228104.

5 C. Dellago, P. G. Bolhuis and P. L. Geissler, Adv. Chem. Phys., 2002, 123, 1.

6 C. Dellago and P. G. Bolhuis, Advanced Computer Simulation Approaches for Soft Matter Sciences III, Springer-Verlag, Berlin, Heidelberg, 2009, vol. 221, p. 167.

7 P. G. Bolhuis and C. Dellago, Eur. Phys. J.: Spec. Top., 2015, 2409.

8 A. K. Faradjian and R. Elber, J. Chem. Phys., 2004, 120, 10880-10889.

9 A. Ma and A. R. Dinner, J. Phys. Chem. B, 2005, 109, 6769-6779.

10 W. E, W. Ren and E. Vanden-Eijnden, Chem. Phys. Lett., 2005, 413, 242-247.

11 R. B. Best and G. Hummer, Proc. Natl. Acad. Sci. U. S. A., 2005, 102, 6732-6737.

12 P. L. Geissler, C. Dellago and D. Chandler, J. Phys. Chem. B, 1999, 103, 3706.

13 W. Lechner, C. Dellago and P. G. Bolhuis, Phys. Rev. Lett., 2011, 106, 085701.

14 B. Peters and B. L. Trout, J. Chem. Phys., 2006, 125, 054108.

15 G. T. Beckham and B. Peters, J. Phys. Chem. Lett., 2011, 2, 1133.

16 S. Jungblut, A. Singraber and C. Dellago, Mol. Phys., 2013, 111, 3527.

17 C. Leitold, W. Lechner and C. Dellago, J. Phys.: Condens. Matter, 2015, 27, 194126.

18 J. Daru and A. Stirling, J. Chem. Theory Comput., 2014, 10, 1121-1127.

19 C. H. Bennett, in Molecular Dynamics and Transition State Theory: The Simulation of Infrequent Events, 1977, ch. 5, pp. 63-97.

20 D. Chandler, J. Chem. Phys., 1978, 68, 2959-2970.

21 T. S. van Erp and P. G. Bolhuis, J. Comput. Phys., 2005, 205, 157-181.

22 L. Rowland, A. C. L. da Costa, D. R. Galbraith, R. S. Oliveira, O. J. Binks, A. A. R. Oliveira, A. M. Pullen, C. E. Doughty, D. B. Metcalfe, S. S. Vasconcelos, L. V. Ferreira, Y. Malhi, J. Grace, M. Mencuccini and P. Meir, Nature, 2015, 528, 119-122.

23 T. D. Wheeler and A. D. Stroock, Nature, 2008, 455, 208-212.

24 O. Vincent, P. Marmottant, P. A. Quinto-Su and C.-D. Ohl, Phys. Rev. Lett., 2012, 108, 184502.

25 S. N. Patek and R. L. Caldwell, J. Exp. Biol., 2005, 208, 3655-3664.

26 X. Noblin, N. O. Rojas, J. Westbrook, C. Llorens, M. Argentina and J. Dumais, Science, 2012, 335, 1322.

27 C. E. Brennen, Cavitation and Bubble Dynamics, Oxford University Press, New York, 1995.

28 P. Kumar and R. Saini, Renewable Sustainable Energy Rev., 2010, 14, 374-383.

29 G. Menzl, M. A. Gonzalez, P. Geiger, F. Caupin, J. L. F. Abascal, C. Valeriani and C. Dellago, 2016, arXiv:1606.03392.

30 J. L. F. Abascal and C. Vega, J. Chem. Phys., 2005, 123, 234505.

31 K. Davitt, A. Arvengas and F. Caupin, EPL, 2010, 90, 16002. 
32 M. A. Gonzalez, G. Menzl, J. L. Aragones, P. Geiger, F. Caupin, J. L. F. Abascal, C. Dellago and C. Valeriani, J. Chem. Phys., 2014, 141, $18 \mathrm{C} 511$.

33 S. Duane, A. Kennedy, B. Pendleton and D. Roweth, Phys. Lett. B, 1987, 195, 216-222.

34 B. Mehlig, D. W. Heermann and B. M. Forrest, Phys. Rev. B: Condens. Matter Mater. Phys., 1992, 45, 679-685.

35 G. Torrie and J. Valleau, J. Comput. Phys., 1977, 23, 187-199.

36 H. C. Andersen, J. Chem. Phys., 1980, 72, 2384-2393.

37 S. Nosé, J. Chem. Phys., 1984, 81, 511.

38 W. G. Hoover, Phys. Rev. A: At., Mol., Opt. Phys., 1985, 31, 1695.

39 H. Kamberaj, R. Low and M. Neal, J. Chem. Phys., 2005, 122, 224114.

40 T. Miller III, M. Eleftheriou, P. Pattnaik, A. Ndirango, D. Newns and G. Martyna, J. Chem. Phys., 2002, 116, 8649.

41 G. Martyna, M. Tuckerman, D. Tobias and M. Klein, Mol. Phys., 1996, 87, 11171157. 